> La biopsie hépatique est actuellement un examen indispensable à la prise en charge des patients atteints d'hépatite chronique C. Elle permet d'évaluer l'intensité des lésions hépatiques, en particulier de la fibrose, de guider le choix thérapeutique et d'évaluer l'efficacité du traitement. Il s'agit cependant d'un examen agressif qui peut être un obstacle à la prise en charge des patients. Le développement de marqueurs sériques biochimiques fiables, reproductibles et de valeur prédictive élevée, pourrait représenter une alternative très utile en permettant de diminuer le nombre de biopsies hépatiques. <

\section{Biopsie du foie contre \\ prise de sang \\ pour le suivi \\ de l'hépatite C ?}

Thierry Poynard, Vlad Ratziu, Joseph Moussalli, Corinne Regimbeau, Vincent di Martino, Yves Benhamou, Rob Myers, Françoise Imbert-Bismut

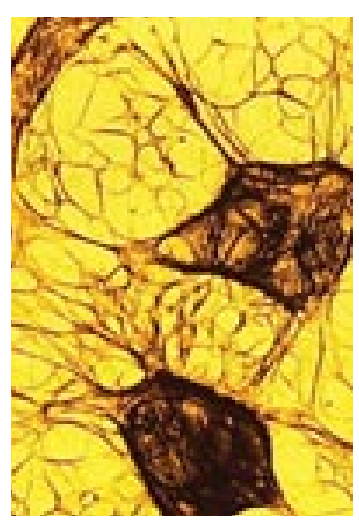

Service d'hépatogastroentérologie, Service de Biochimie, Groupe MULTIVIRC, Groupe Hospitalier Pitié-Salpêtrière, 47, boulevard de l'Hôpital, 75651 Paris Cedex 13, France.
L'évaluation de l'état histologique du foie est considérée comme une donnée indispensable pour décider du traitement et du suivi des sujets contaminés par le virus de l'hépatite C (VHC) [1]. La biopsie hépatique n'est toutefois pas un examen anodin [2] et, souvent vécue par les sujets contaminés par le VHC comme un examen agressif [3], elle peut se révéler un obstacle à la prise en charge efficace de l'hépatite $C$. Une enquête récente (Réseau Hépatite C-Auvergne, chez 1177 généralistes) a révélé que la biopsie hépatique était refusée par $59 \%$ des patients contaminés par le VHC et que $22 \%$ des médecins interrogés partageaient la même crainte de la biopsie [3]. En dehors de ces enquêtes d'opinion, les études portant sur les complications de la biopsie montrent qu'une douleur est signalée chez un tiers des sujets, qu'une complication sévère existe dans 3 cas sur 1000 et un décès dans 3 cas sur 10000 [2,4].

L'utilisation de marqueurs non agressifs de l'état histologique du foie pourrait donc s'avérer très importante pour assurer une prise en charge plus efficace de cette maladie. Dans cet objectif, nous avons mis au point une combinaison de tels marqueurs qui pourrait permettre de diminuer par deux le nombre de biopsies du foie chez les sujets contaminés par le VHC [5].

\section{A quoi sert la biopsie hépatique dans la prise en charge de l'hépatite C?}

La biopsie hépatique permet tout d'abord d'estimer la gravité de l'hépatite $C$. La mortalité de cette maladie est liée à la survenue des complications de la cirrhose, cancer primitif du foie, hémorragie digestive et insuffisance hépatocellulaire. Plus la cirrhose se développe rapidement, plus le risque de mortalité est élevé. L'estimation de la vitesse de progression des lésions hépatiques vers la cirrhose (ou vitesse de progression de la fibrose) est donc le meilleur indice de gravité de l'infection chronique par le VHC. Une classification simple et reproductible des stades de fibrose est la classification METAVIR (acronyme du groupe de pathologistes français l'ayant validé) qui comprend cinq stades : F0 pas de fibrose, Fl fibrose limitée à l'espace porte, F2 fibrose avec quelques septums, F3 nombreux septums et F4 cirrhose. Quand la durée de l'infection est connue, il est donc possible d'estimer la vitesse moyenne de progression de la fibrose. La biopsie hépatique permet également d'estimer le grade d'activité des lésions, c'està-dire l'intensité de la nécrose cellulaire et de l'inflammation locale ( $\mathrm{A} 0$ pas d'activité, $\mathrm{Al}$ activité minime, $\mathrm{A} 2$ 
activité modérée, $A 3$ activité sévère). Ce paramètre est cependant moins prédictif de la morbidité et de la mortalité que le stade de fibrose car, dans de nombreux cas, un grade d'activité élevé ne s'accompagne pas d'une évolution vers la cirrhose. Ce phénomène de tolérance à la nécrose sans fibrose n'est pas clairement expliqué mais pourrait être dû à l'intervention de facteurs antifibrosants, comme les collagénases.

Un autre intérêt de la biopsie hépatique des patients infectés par le VHC est de préciser s'il existe d'autres facteurs d'agression comme l'alcool, l'infection par le virus de l'hépatite $B$, la prise de médicaments hépatotoxiques, ou la présence des maladies métaboliques (diabète, hyperlipémie, hémochromatose). L'étude histologique peut en effet permettre de mieux faire la part entre la toxicité de ces différents facteurs.

\section{En quoi consistent les marqueurs biochimiques de fibrose?}

Le premier point qu'il paraît important de souligner est qu'aucun test sanguin, pris isolément, ne possède des valeurs prédictives négatives ou positives suffisantes pour se substituer à la biopsie hépatique, en particulier pour le diagnostic de fibrose modérée (F2) [5]. Les tests les moins mauvais sont la détermination du taux de prothrombine et du nombre de plaquettes, et le dosage de l'acide hyaluronique et du propeptide du collagène III $[6,7]$. Quant à l'échographie du foie, elle ne permet pas de prédire les stades précoces de fibrose et, même en cas de cirrhose, sa sensibilité ne dépasse pas $50 \%$.

Afin de déterminer la meilleure combinaison de marqueurs simples, robustes, facilement dosables et automatisables, nous avons procédé de façon prospective avec une première phase d'identification et de combinaison des marqueurs suivie d'une seconde phase de validation. Parmi une quinzaine de marqueurs potentiels de fibrose hépatique, cinq ont été sélectionnés et validés ${ }^{\star}$. L'index de fibrose combine le dosage dans le sang de l' $\alpha 2$-macroglobuline, l'haptoglobine, l'apolipoprotéine Al, la bilirubine totale, la $\gamma$-glutamyl transpeptidase, dont les concentrations sont ajustées selon l'âge et le sexe de la personne (Tableau I) [5]. nèse [11]. L' $\alpha 2 \mathrm{~m}$, inhibe les protéinases et peut donc augmenter la fibrose en inhibant le catabolisme des protéines de la matrice extracellulaire, par exemple en diminuant l'activité des collagénases [12].

\section{Haptoglobine}

L'haptoglobine est aussi une protéine synthétisée par le foie. Contrairement à l' $\alpha 2 \mathrm{~m}$, sa concentration diminue en cas de fibrose [13-14], et ce de façon indépendante de l'hémolyse ou de l'insuffisance hépatique. Cet effet opposé de la fibrose sur l' $\alpha 2 \mathrm{~m}$ et l'haptoglobine pourrait être expliquée par des régulations inverses de l'hepatocyte growth factor (HGF) et du transforming growth factor (TGF) $\beta 1$ pendant la fibrogenèse et l'inflammation $[14,15]$. De façon surprenante, nous avons constaté que la fibrose hépatique est associée à une augmentation de l'HGF et à une diminution du TGF $\beta$ l, et non pas à une augmentation comme on pouvait s'y attendre [5]. On peut envisager, comme cela a été montré au cours de la fibrose expérimentale, que ce soit I'HGF qui soit responsable de la diminution de l'expression du TGF $\beta 1$ [15]. De même, il a été montré qu'in vitro l'HGF stimule la synthèse d' $\alpha 2 \mathrm{~m}$ et diminue la synthèse d'haptoglobine [15-17].

\section{Apolipoprotéine A1}

L'apolipoprotéine $A l(A p o A l)$, synthétisée par les hépatocytes, est le composant protéique des particules HDL (high density lipoprotein) dont le rôle est le transport du cholestérol. En cas de fibrose hépatique, il existe d'une part une diminution de la transcription de l'ApoAl et, d'autre part, une rétention de la protéine sécrétée par les composants de la matrice extracellulaire déposés en excès, en particulier la fibronectine. [18]. Ceci est probablement la cause de la diminution de la concentration sérique d'ApoAl.

\section{Bilirubine totale}

La bilirubine est un pigment protéique provenant de la dégradation de l'hémoglobine. Elle est normalement épurée du sang par le foie qui l'évacue dans la bile. $\varepsilon$ n

\section{a2-macroglobuline}

L' $\alpha 2$-macroglobuline $(\alpha 2 \mathrm{~m})$ est une protéine synthétisée par le foie (hépatocytes, cellules stellaires, granulomes) au cours des processus inflammatoires $[8,9]$. Elle a surtout été étudiée dans les phases aiguës de l'inflammation chez l'animal et peu en situation d'inflammation chronique. Cependant, on sait que sa concentration sérique augmente avec la fibrose [10], une augmentation liée à l'activation des cellules stellaires au cours de la fibroge-

* Des détails et des exemples sont disponibles sur le site www. fibrotest.com

\begin{tabular}{|c|c|c|c|}
\hline & \multicolumn{2}{|c|}{$\begin{array}{c}\text { Concentration } \\
\text { normale }\end{array}$} & \multirow[t]{2}{*}{$\begin{array}{c}\text { Évolution pendant } \\
\text { la fibrose }\end{array}$} \\
\hline & Femmes & Hommes & \\
\hline$\alpha 2$-macroglobuline $\mathrm{g} / \mathrm{I}$ & $1,6-4$ & $1,4-3,3$ & Augmentation \\
\hline Haptoglobine $\mathrm{g} / \mathrm{I}$ & \multicolumn{2}{|c|}{$0,35-2$} & Diminution \\
\hline Apoliproprotéine Al g/l & \multicolumn{2}{|c|}{$1,2-1,7$} & Diminution \\
\hline$\gamma$-glutamyl transpeptidase $\mathrm{U} / \mathrm{I}$ & $7-32$ & $11-49$ & Augmentation \\
\hline Bilirubine totale $\mu \mathrm{mol} / \mathrm{I}$ & \multicolumn{2}{|c|}{$1-21$} & Augmentation \\
\hline
\end{tabular}

Tableau I. Marqueurs biochimiques utilisés dans le test de fibrose. 


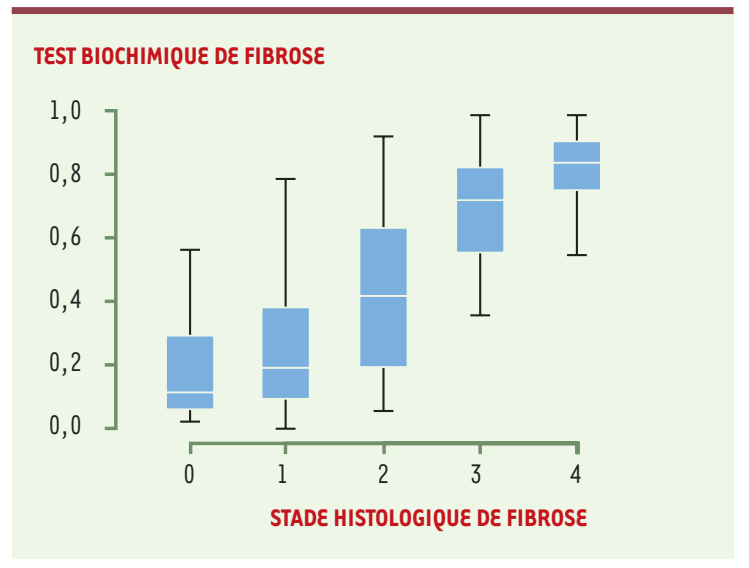

Figure 1. Combinaison de 5 marqueurs biochimiques (Fibrotest) et stades de fibrose. II existe une augmentation du test de fibrose en fonction des stades de fibrose ( 0 = rien; 4 = cirrhose $)$.

cas de fibrose, la concentration plasmatique de bilirubine augmente.

\section{$\gamma$-glutamyl transpeptidase}

La $\gamma$-glutamyl transpeptidase ( $\gamma$ GT) est une enzyme synthétisée par les hépatocytes. Sa concentration augmente en cas de fibrose, mais les mécanismes impliqués sont inconnus. Dans notre expérience, cette augmentation est associée à celle de l'HGF mais est indépendante de celle des transaminases et de la bilirubine. Une hypothèse serait, qu'en dehors même de toute cholestase, cet effet soit dû à une stimulation de la synthèse de l'epidermal growth factor au cours de la fibrogenèse [19].

\section{Interprétation et limites des marqueurs biochimiques de fibrose}

La combinaison de ces cinq marqueurs a permis de définir, par une régression logistique, un index de fibrose compris entre 0,00 et 1,00 . Quand cet index reste très bas (de 0,00 à 0,10), la probabilité d'avoir une fibrose significative dans le foie est très faible, proche de zéro. Quand l'index est compris entre 0,60 et 1,00, la probabilité de fibrose est supérieure à $90 \%$. Entre 0,10 et 0,60 , les valeurs prédictives sont inférieures à $90 \%$ et il est conseillé de pratiquer une biopsie (Figure 1).

\section{Validation dans d'autres populations de patients contaminés par le VHC}

Afin de vérifier la reproductibilité de cette combinaison de marqueurs, nous avons analysé de façon rétrospective le sérum de 165 patients inclus dans un essai randomisé multicentrique visant à évaluer l'efficacité d'un traitement par l'interféron. Dans cette étude, deux biopsies hépatiques ont été effectuées, avant et après le traitement, et la valeur du test de fibrose a été comparée à celle de l'acide hyaluronique à partir de prélèvements sanguins effectués au même moment que les biopsies [20]. Les résultats montrent que l'index de fibrose produit les mêmes valeurs prédictives pour la fibrose et l'activité en comparaison avec l'étude précédente. De plus, il existe une très bonne corrélation entre la réponse virologique et les marqueurs biochimiques de fibrose (Figure 2). La valeur diagnostique de la combinaison des cinq marqueurs biochimiques se révèle supérieure à celle de l'acide hyaluronique que ce soit avant, pendant, ou après le traitement.

\section{A}

FIBROTEST

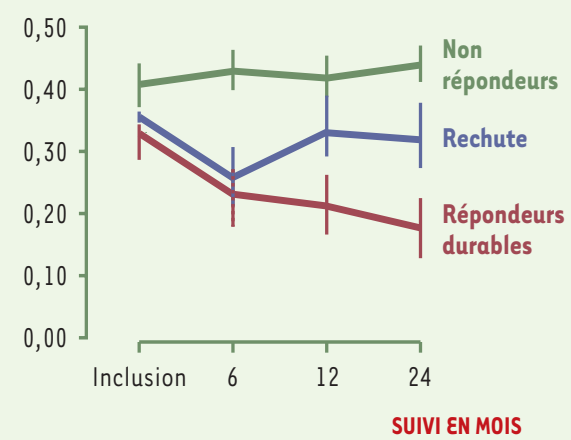

B

ACIDE HYALURONIPUE

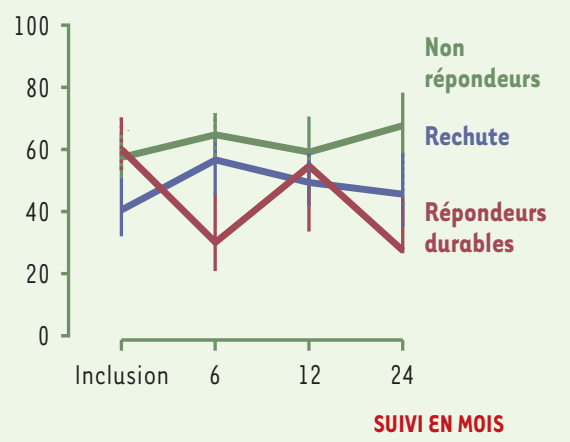

Figure 2. Évaluation longitudinale de la combinaison biochimique (Fibrotest) (A) et de l'acide hyaluronique (B) en fonction de la réponse virologique. Répondeurs durables = disparition du virus sous traitement par interféron.

Pour l'instant, ce test biochimique de fibrose fait encore partie du domaine de la recherche clinique et il apparaît indispensable de poursuivre des études prospectives afin de déterminer dans quelles conditions il pourra se substituer à la biopsie hépatique. Les résultats obtenus indiquent que ce test pourrait déjà permettre de simplifier l'abord initial et le suivi des patients après leur traitement. Des résultats préliminaires suggèrent également son utilité dans d'autres maladies fibrosantes comme I'hépatite B ou les hépatopa- 
thies alcooliques. La simplification de l'estimation de la gravité des atteintes histologiques du foie est un impératif compte tenu de la disponibilité de traitements maintenant très efficaces. $\diamond$

\section{SUMMARY}

Could biochemical markers of liver fibrosis reduce the number of liver biopsies?

Liver biopsy is actually essential for the management of patients infected by hepatitis C virus. It is necessary to grade and stage hepatitis and fibrosis, and make decision about treatment. However, liver biopsy is aggressive and can be a limitation for patients management. With a combination of five basic serum biochemical markers for diagnosis of fibrosis, high positive and negative predictive values of important fibrosis can be obtained, suggesting that this index of fibrosis could be used to substantially reduce the number of liver biopsies. $\diamond$

\section{RÉFÉRENCES}

1. Consensus statement. EASL International Consensus Conference on Hepatitis C.J Hepatol 1999 ; 30 : 956-61.

2. Cadranel JF, Rufat P, Degos F. Practices of liver biopsy in France: results of a prospective nationwide survey. Hepatology 2000 ; 32 : 477-81.

3. Rayssiguier R, Bonny C, Abergel $A$, et al. Pratiques et attentes des médecins généralistes en matière d'hépatite $C$ dans la région Auvergne. Gastroenterol Clin Biol 2001 ; 25 : A67 (abstract).

4. Poynard T, Ratziu V, Bedossa P. Appropriateness of liver biopsy. Can J Gastroenterol 2000 ; 14 : 543-8.

5. Imbert-Bismut F, Ratziu V, Pieroni L, et al. Biochemical markers of liver fibrosis in patients with hepatitis $C$ virus infection : a prospective study. Lancet 2001 ; 357 : 1069-75.

6. Guechot J, Laudat A, Loria A, Serfaty L, Poupon R, Giboudeau J. Diagnostic accuracy of hyaluronan and type III procollagen aminoterminal peptide serum assays as markers of liver fibrosis in chronic viral hepatitis $\mathrm{C}$ evaluated by ROC curve analysis. Clin Chem 1996; 42 : 558-63.

7. Oberti F, Valsesia $\varepsilon$, Pilette $C$, et al. Noninvasive diagnosis of hepatic fibrosis or cirrhosis. Gastroenterology 1997 ; 113 : 1609-16.

8. Tiggelman AM, Boers W, Moorman AF, et al. Localization of alpha 2macroglobulin protein and messenger RNA in rat liver fibrosis: evidence for the synthesis of alpha 2macroglobulin within Schistosoma mansoni egg granulomas. Hepatology 1996 ; 23 : 1260-7.

9. Tiggelman AM, Linthorst $C$, Boers W, Brand HS, Chamuleau RA.

Transforming growth factorbeta-induced collagen synthesis by human liver myofibroblasts is inhibited by alpha2-macroglobulin. J Hepatol 1997 ; 26 : 1220-8.

10. Naveau S, Poynard T, Benattar C, Bedossa P, Chaput JC. Alpha-2macroglobulin and hepatic fibrosis. Diagnostic interest. Dig Dis Sci 1994 ; 39 : 2426-32.

11. Meisse D, Renouf $S$, Husson A, Lavoinne A. Cell swelling increased the alpha2macroglobulin gene expression in cultured rat hepatocytes. FEBS Lett 1998 ; 422 : 346-8.

12. Kawser CA, Iredale JP, Winwood PJ, Arthur MJ. Rat hepatic stellate cell expression of alpha2macroglobulin is a feature of cellular activation: implications for matrix remodelling in hepatic fibrosis. Clin Sci 1998 ; 95 : 179-86.

13. Bacq $Y$, Schillio $Y$, Brechot JF, De Muret A, Dubois F, Metman EH. Decrease of haptoglobin serum level in patients with chronic viral hepatitis C. Gastroenterol Clin Biol 1993 ; 17 : 364-9.

14. Louagie HK, Brouwer JT, Delanghe JR, De Buyzere ML, Leroux-Roels GG. Haptoglobin polymorphism and chronic hepatitis C. J Hepatol 1996 ; 25 : 10-4.

15. Ueki T, Kaneda Y, Tsutsui $H$, et al. Hepatocyte growth factor gene therapy of liver cirrhosis in rats. Nat Med 1999 ; 5 : 226-30.

16. Guillen MI, Gomez-Lechon MJ, Nakamura T, Castell JV. The hepatocyte growth factor regulates the synthesis of acute-phase proteins in human hepatocytes: divergent effect on interleukin-6stimulated genes. Hepatology 1996 ; 23 : 1345-52.

17. Moshage H. Cytokines and the hepatic acute phase response. J Pathol 1997 ; $181:$ 257-66.

18. Paradis V, Laurent $A$, Mathurin P, et al. Role of liver extracellular matrix in transcriptional and posttranscriptional regulation of apolipoprotein A-I by hepatocytes. Cell Mol Biol 1996 ; 42 : 525-34.

19. Edwards AM, Lucas CM, Baddams HM. Modulation of gamma-glutamyltranspeptidase in normal rat hepatocytes in culture by cell density, epidermal growth factor and agents which alter cell differentiation. Carcinogenesis $1987 ; 8$ : 1837-42.

20. Poynard T, Imbert-Bismut F, Ratziu V, et al. Biochemical markers of liver fibrosis in patients infected by hepatitis $C$ virus: longitudinal validation in a randomized trial. J Viral Hepatitis 2002 (sous presse). 